ORIGINAL ARTICLE

\title{
Changes in Anterior Chamber Depth and Angle Before and After Phacoemulsification of Intumescent Cataract
}

\author{
Mohamed Anas Hussein I, Moustafa Abdullah Salamah I, Medhat Mohamed Shawky I, Kamal \\ Abdel-Moniem Solaiman ${ }^{\text {I* }}$ \\ I: Department of Ophthalmology, Faculty of medicine, Zagazig university, Zagazig, Egypt
}

* Corresponding Author
Kamal Abdel-Moniem
Solaiman
Kams_8866@yahoo.com

Submit Date 2019-07-21

Accept Date 2019-07-29
ABSTRACT
Background: To use the Sirius imaging system to precisely evaluate the changes in the anterior chamber depth and angle before and after phacoemulsification of intumescent cataract in eyes with different axial lengths. Methods: A prospective study that included consecutive patients with intumescent cataract in one eye. All eyes were subjected to full ophthalmic examination, IOP, gonioscopy, ocular ultrasonography (A and B scans), IOL master and axial length measurement. Anterior segment imaging of both eyes using Sirius imaging system to determine the anterior chamber angle (ACD) and anterior chamber angle (ACA). Eyes were classified into three groups according to their axial length (AXL); Group I: axial length < $22 \mathrm{~mm}$, Group II: axial length $22-24.5 \mathrm{~mm}$, and Group III: axial length > $24.5 \mathrm{~mm}$. Postoperative examinations were performed at 1 day, 1 week, 1 month and 3 months. Results: The study enrolled 32 eyes; Group I included 11 eyes, Group II included 11 eyes and Group III included 10 eyes. Before surgery, the mean ACD was $2.03 \pm 0.46 \mathrm{~mm}$ in Group I, $2.2 \pm 0.42 \mathrm{~mm}$ in Group II and $2.62 \pm 0.37 \mathrm{~mm}$ in Group III $(\mathrm{P}<0.05)$. The mean ACA width was $18.36 \pm 3.64^{\circ}$ in Group I, $20.27 \pm 4.86^{\circ}$ in Group II and $26.8 \pm 7.93^{\circ}$ in Group III $\quad(\mathrm{P}<0.05)$. The mean IOP was $22.73 \pm 1.74 \mathrm{mmHg}$ in Group I, $14.45 \pm 1.13 \mathrm{mmHg}$ in Group II and $13.7 \pm 0.95 \mathrm{mmHg}$ in Group III (P < $0.05)$. After surgery, all the investigated parameters have been statistically significantly changed when compared to the preoperative values $(\mathrm{P}<0.05)$. The mean ACD was $3.44 \pm 0.28 \mathrm{~mm}$ with a mean relative change $(80.19 \%)$ in Group I, $3.3 \pm 0.39 \mathrm{~mm}$ with a mean relative change ( $52.76 \%$ ) in Group II and $3.33 \pm 0.32 \mathrm{~mm}$ with a mean relative change ( 30.76\%) in Group III (P < $0.05)$. The mean ACA width was $32.36 \pm 3.72^{\circ}$ with a mean relative change $(82.35 \%)$ in Group I, $31.64 \pm 2.62^{\circ}$ with a mean relative change $(58.82 \%)$ in Group II and $32.1 \pm 7.22^{\circ}$ with a mean relative change (20\%) in Group III (P $<0.05)$. The mean IOP was $15.82 \pm 1.33 \mathrm{mmHg}$ with a mean relative change ( $30 \%$ ) in Group I, $12.36 \pm 1.33 \mathrm{mmHg}$ with a mean relative change $(14.29 \%)$ in Group II and $12 \pm 1.25 \mathrm{mmHg}$ with a mean relative change ( $7.69 \%$ ) in Group III ( $\mathrm{P}<0.05)$. Conclusion: Intumescent cataract induces significant reductions in the ACD and ACA and phacomorphic glaucoma could be induced in short eyes. Phacoemulsification significantly increases the ACD and ACA width and could be an effective treatment of phacomorphic glaucoma. Measuring the axial length in eyes with intumescent cataract could predict eyes susceptible to phacomorphic glaucoma.

Key words: Intumescent cataract - Anterior chamber depth - Anterior chamber angle - Sirius imaging system)

\section{INTRODUCTION}

I ntumescent cataract is characterized by swelling of mature or hypermature cataractous lens with increase of its anteroposterior diameter and subsequent shallowing of the anterior chamber and narrowing of its angle [1,2]. This could induce elevation of intraocular pressure due to 
phacomorphic angle closure with or without pupillary block (1). The severity of these anterior segment changes caused by a swollen lens vary with variation of the axial length of the globe [3]. Knowing the axial length of an eye with intumescent cataract could enable us to properly predict the severity of the risk that could be induced by a swollen lens.

The machines used for anterior segment imaging as OCT, Scheimpflug camera and UBM can provide a precise quantitative assessment of the anterior segment structures $[4,5,6]$, and can be used to assess the changes induced by intumescent cataract before and after phacoemulsification. Sirius 3D Rotating Camera - Topography system is using the Scheimpflug camera for anterior segment imaging and has excellent repeatability and reproducibility for measuring the anterior segment parameters $[7$, 8]. The aim of this study is to use the Sirius anterior segment imaging to precisely evaluate the changes in the anterior chamber depth and angle before and after phacoemulsification of intumescent cataract in eyes with different axial lengths.

\section{METHODS}

After obtaining approval from the scientific committee of ophthalmology department and the institutional review board (IRB) of faculty of medicine Zagazig University. Written informed consent was obtained from all participants. The work has been carried out in accordance with The Code of Ethics of the World Medical Association (Declaration of Helsinki) for studies involving humans.

A prospective study that included consecutive patients with intumescent cataract who attended the Department of Ophthalmology in Zagazig University, during the period from august 2018 to march 2019. The study included adult patients with intumescent cataract and scheduled for phacoemulsification. Only one eye per patient was included. Intumescent cataract was diagnosed when there is a swollen cataractous lens and shallow anterior chamber when compared to the fellow eye, with or without elevation of IOP. Exclusion criteria were no perception of light, traumatic cataract, subluxation of the lens, previous intraocular surgery, extended anterior capsulorehxis and posterior capsular tear.

All eyes were subjected to full ophthalmic examination including visual acuity, IOP (applanation tonometer), slit lamp examination of anterior segment, gonioscopy, ocular ultrasonography (A and B scans), IOL master and axial length measurement. Anterior segment imaging of both eyes using Sirius 3D Rotating Camera - Topography system (Costruzioni Strumenti Oftalmici, Florence, Italy) with the pupil not dilated to determine the following anterior segment parameters:

- ACD: a line from the centre of the inner corneal surface perpendicular to the plane of the anterior surface of the iris.

- Relative change in the $\mathrm{ACD}=$ postoperative $\underline{\mathrm{ACD}}$ - preoperative ACD $\times 100$

\section{preoperative ACD}

- ACA: the mean of the trabecular - iris angle measurements at 2 nasal and 2 temporal points.

- Relative change in $\mathrm{ACA}=$ postoperative $\mathrm{ACA}$ - preoperative ACA $\times 100$

\section{preoperative ACA}

Eyes were classified into three groups according to their axial length; Group I: axial length < $22 \mathrm{~mm}$, Group II: axial length $22-$ $24.5 \mathrm{~mm}$, and Group III: axial length > 24.5 $\mathrm{mm}$. All the examinations and interpretation of the results were done by the same investigator. Eyes with IOP $\geq 21 \mathrm{mmHg}$ received anti glaucoma medications before surgery. Cataract surgery was performed under local anaesthesia with or without sedation. Trypan blue was used for staining of the anterior capsule, and a foldable IOL was implanted in the bag in all eyes. Eyes complicated by extension of the anterior capsulorrhexis (Argentina flag sign) and those with posterior capsular tear were excluded from the study. Postoperative examinations were performed at 1 day, 1 week, 1 month and 3 months. Postoperative anterior segment imaging was done at 3 months.

\section{Statistical analysis}

Statistical analyses of data were performed using Data analysis was performed using the software SPSS (Statistical Package 
for the Social Sciences) version 20. Quantitative variables were described using their means and standard deviations. Categorical variables were described using their absolute frequencies and were compared using Chi square test. Kolmogorov-Smirnov (distribution-type) and Levene (homogeneity of variances) tests were used to verify assumptions for use in parametric tests. To compare means of the same group before and after phototherapy, paired sample $t$ test was used for normally distributed data. To compare means of more than two groups, two way ANOVA test (for normally distributed data) and Kruskal Wallis test (for not normally distributed data) were used. The level statistical significance was set at $5 \%$ $(P<0.05)$. Highly significant difference was present if $\mathrm{p} \leq 0.001$

\section{RESULTS}

The study included 33 consecutive patients with unilateral intumescent cataract. One eye was excluded from the study due to extended anterior capsulorehxis during surgery and the actual number of eyes enrolled in the study was 32 eyes. The mean age of all patients was $63.53 \pm 9.12$ years (Range: 42 - 82 years); $43.8 \%$ were males and $56.2 \%$ were females. Group I included 11 eyes, Group II included 11 eyes and Group III included 10 eyes. The demographic data of the 3 groups were illustrated in Table (1).

Before surgery, the mean ACD was $2.03 \pm 0.46 \mathrm{~mm}$ in Group I, $2.2 \pm 0.42 \mathrm{~mm}$ in Group II and 2.62 $\pm 0.37 \mathrm{~mm}$ in Group III (P $<0.05)$. The mean ACA width was $18.36 \pm$ $3.64^{\circ}$ in Group I, $20.27 \pm 4.86^{\circ}$ in Group II and $26.8 \pm 7.93{ }^{\circ}$ in Group III $(\mathrm{P}<0.05)$. The mean IOP was $22.73 \pm 1.74 \mathrm{mmHg}$ in Group I, $\quad 14.45 \pm 1.13 \mathrm{mmHg}$ in Group II and $13.7 \pm 0.95 \mathrm{mmHg}$ in Group III $(\mathrm{P}<0.05)$, Table (2). The mean preoperative IOP was higher in the affected eyes than in their fellow eyes in all groups but was statistically significant only in Group I, in which IOP was elevated $>21 \mathrm{mmHg}$ in 5 eyes $(45.5 \%)$ that received medications to lower the IOP. None of the eyes in Group II and Group III had IOP $\geq 21 \mathrm{mmHg}$. None of the eyes in all groups had peripheral anterior synechiae before or after surgery.

After surgery, all the investigated parameters have been statistically significantly changed when compared to the preoperative values $(\mathrm{P}$ $<0.05)$. The mean ACD was $3.44 \pm 0.28 \mathrm{~mm}$ with a mean relative change $(80.19 \%)$ in Group I, $3.3 \pm 0.39 \mathrm{~mm}$ with a mean relative change (52.76\%) in Group II and 3.33 \pm 0.32 $\mathrm{mm}$ with a mean relative change $(30.76 \%)$ in Group III $(\mathrm{P}<0.05)$. The mean ACA width was $32.36 \pm 3.72{ }^{\circ}$ with a mean relative change $(82.35 \%)$ in Group I, $31.64 \pm 2.62^{\circ}$ with a mean relative change $(58.82 \%)$ in Group II and $32.1 \pm 7.22^{\circ}$ with a mean relative change $(20 \%)$ in Group III $(\mathrm{P}<0.05)$. The mean IOP was $15.82 \pm 1.33 \mathrm{mmHg}$ with a mean relative change $(30 \%)$ in Group I, $12.36 \pm 1.33 \mathrm{mmHg}$ with a mean relative change (14.29\%) in Group II and $12 \pm 1.25$ $\mathrm{mmHg}$ with a mean relative change $(7.69 \%)$ in Group III ( $\mathrm{P}<0.05)$, Table (5). No eye had postoperative IOP elevation in all groups.

Table 1. Demographic data and mean axial length in the three groups

\begin{tabular}{|c|l|l|l|}
\hline $\begin{array}{c}\text { Number of eyes: } \\
\text { Males }\end{array}$ & Group I & Group II & Group III \\
$\quad$ Females & $3(27.7)$ & $7(63.6)$ & $3(30)$ \\
Mean age (years): & $63.64 \pm 9.94$ & $4(36.4)$ & $7(70)$ \\
$\quad$ Range & $42-74$ & $64.55 \pm 10.53$ & $62.3 \pm 7.09$ \\
Mean axial length & & $52-82$ & $53-72$ \\
$\quad$ Affected eye & $21.75 \pm 0.21$ & $23.02 \pm 0.38$ & $25.18 \pm 0.67$ \\
Fellow eye & $21.94 \pm 0.54$ & $22.96 \pm 0.54$ & $24.65 \pm 0.72$
\end{tabular}


Table 2. The preoperative data in both the affected and fellow eyes in the three groups

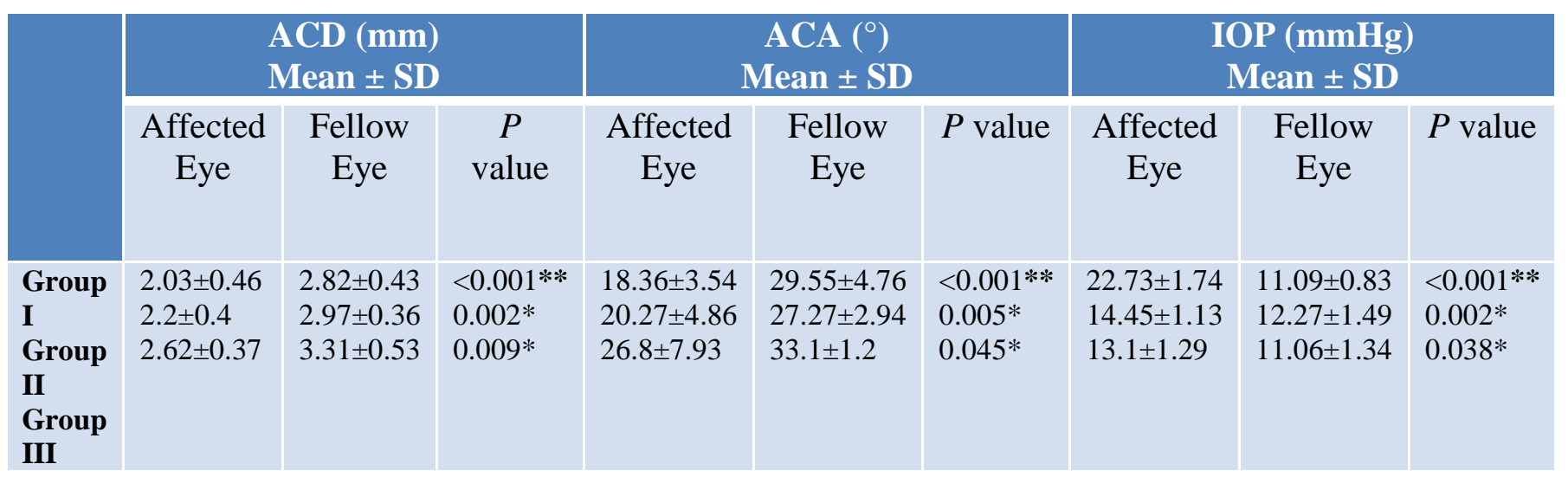

Table 3. Mean preoperative and mean postoperative ACD and the relative change in the 3 groups

\begin{tabular}{l|l|l|l|}
\multicolumn{1}{c|}{ ACD } & \multicolumn{1}{c|}{ Group I } & \multicolumn{1}{c|}{ Group II } & \multicolumn{1}{c|}{ Group III } \\
Mean preoperative & $2.03 \pm 0.46$ & $2.2 \pm 0.42$ & $2.62 \pm 0.37$ \\
Mean postoperative & $3.44 \pm 0.28$ & $3.3 \pm 0.39$ & $3.33 \pm 0.32$ \\
Relative change $(\%)$ & 80.19 & 52.76 & 30.76 \\
$\boldsymbol{P}$ value & $<0.001 * *$ & $<0.001 * *$ & $<0.001 * *$
\end{tabular}

Table 4. Mean preoperative and mean postoperative ACA and the relative change in the 3 groups

\begin{tabular}{|l|l|l|l|}
\multicolumn{1}{c|}{ ACA } & \multicolumn{1}{c|}{ Group I } & \multicolumn{1}{c|}{ Group II } & \multicolumn{1}{c|}{ Group III } \\
\hline Mean preoperative & $18.36 \pm 3.64$ & $20.27 \pm 4.86$ & $26.8 \pm 7.93$ \\
Mean postoperative & $32.36 \pm 3.72$ & $31.64 \pm 2.62$ & $32.1 \pm 7.22$ \\
Relative change & 82.35 & 58.82 & 20 \\
$(\%)$ & $<0.001 * *$ & $<0.001 * *$ & $<0.001 * *$ \\
$P$ value & & &
\end{tabular}

\section{DISCUSSION}

Intumescent cataract is usually due to swelling of the lens in eyes with mature or hypermature cataract. Lens swelling causes forward movement of the iris with or without pupillary block and this could induce elevation of the IOP due to changes in the anterior segment parameters; namely the anterior chamber depth and angle [1,2]. These changes vary between eyes depending mainly upon the axial length of the affected eye. No study has been done before on eyes with intumescent cataract to demonstrate which eye is more liable to develop phacomorphic glaucoma. This study investigates quantitatively the changes in these parameters in eyes with intumescent cataract before and after phacoemulsification. Postoperative anterior segment imaging was done at 3 months to allow enough time for the capsular bag fibrosis to settle down and for the IOL to be stable in position, and consequently the recorded parameters will be more accurate.

Before phacoemulsification, the swollen lens induced a statistical significant reduction in the mean $A C D$ and the mean ACA width of the affected eyes as compared to the fellow non affected eyes, and the difference was highly significant in the short eyes ( $\mathrm{P}<0.001$ for both ACD and ACA) than in the long eyes $(\mathrm{P}=0.009$ for $\mathrm{ACD}$ and 0.045 for ACA). In their study on short eyes (mean AXL: $22.3 \mathrm{~mm}$ ) with phacomorphic glaucoma treated by phacoemulsification and IOL implantation, Lee et al reported a significant increase in the mean ACD from $1.5 \mathrm{~mm}$ preoperative to $2.6 \mathrm{~mm}$ after surgery; which is matching with our results [1]. They did not measure the ACA in their cases as they were using the A-scan ultrasonography which cannot measure the ACA. Another study that used the anterior segment OCT to measure the change in ACD after 
phacoemulsification in short and long eyes, had concluded that the relative increase in ACD was significantly larger in short eyes than in normal or long eyes [3], which matches with our results.

Lin et al in their study on biometry of acute angle closure glaucoma reported that eyes with angle closure glaucoma had a mean ACD $1.8 \pm 0.2 \mathrm{~mm}$ less than in normal eyes, and that the mean AXL was $22.3 \pm 0.8 \mathrm{~mm}$ which was $1.0 \mathrm{~mm}$ shorter than in normal eyes. They found also that relative pupillary block is rare when the AC is deeper than 2.7 $\mathrm{mm}$ [9]. Their findings match with and support the results of our study in which 5 eyes $(45.5 \%)$ in Group I (mean AXL $=22.3$ $\mathrm{mm}$ ) had phacomorphic glaucoma and none of the eyes in Group II and Group III had elevated IOP due to intumescent cataract. This indicates that short eyes with intumescent cataract are highly vulnerable to develop phacomorphic glaucoma and should have urgent phacoemulsification before development of peripheral anterior synechiae or optic nerve damage. Examination of the fellow eye is essential to distinguish phacomorphic glaucoma from primary angle closure glaucoma in which the anterior chamber periphery is shallow in both eyes and the lens is not swollen.

In this study, phacoemulsification of intumescent cataract has resulted in statistically significant increase in the mean ACD and ACA width in the three groups as compared to the preoperative values. The relative change in the mean ACD was larger in Group I than in Group II and in Group III. This could be because the lens thickness to AXL ratio is larger in the short eyes (Group I) than in the normal eyes (Group II) and in long eyes (Group III). A study on ultrasonic evaluation of lens thickness to axial length ratio found that a ratio higher than 0.2 was a risk factor in eyes with angle closure glaucoma [10]. Many studies agreed with the results of this study and demonstrated that phacoemulsification induced changes in the anterior segment parameters, and the most profound postoperative changes in ACD and ACA were observed in eyes with the smallest preoperative ACD and ACA [6, 11, 12].
However, unlike in our study the lens was not swollen in these studies. It is expected that the change in the anterior segment parameters after phacoemulsification will be more prominent in eyes with a swollen lens (intumescent cataract) due to larger lens thickness to AXL ratio than in eyes without lens swelling. Other studies reported change of the preoperative convex iris shape into a flat shape after cataract extraction due to posterior movement of the iris with subsequent increase of ACD. Also, posterior displacement of the iris base following removal of the lens leads to widening of the iridocorneal angle [13,14].

Before phacoemulsification, the mean IOP was higher in the affected eyes than in their fellow eyes in all groups but was statistically significant only in Group I, in which IOP was elevated $>21 \mathrm{mmHg}$ in 5 eyes $(45.5 \%)$. The mean postoperative IOP has been decreased in the three groups as compared to the mean preoperative values, was statistically significant in all groups, and the relative change was higher in Group I than in Group II and Group III. These results indicate that lens swelling could significantly elevate the IOP in short eyes, and that removal of the swollen lens early before formation of peripheral anterior synechiae is enough for IOP reduction to normal levels. These results match with many results that concluded reduction of IOP following lens extraction in normal eyes and in eyes with primary angle closure glaucoma $[1,2,11]$.

\section{CONCLUSION}

Intumescent cataract induces significant reductions in the ACD and ACA and phacomorphic glaucoma could be induced in short eyes. Phacoemulsification significantly increases the ACD and ACA width and could be an effective treatment of phacomorphic glaucoma. Axial length measurement in eyes with intumescent cataract could predict eyes susceptible to phacomorphic glaucoma.

Conflict of Interest: Nothing to declare. Financial Disclosures: Nothing to declare. REFERENCES

1- Lee SJ, Lee CK, Kim WS. Long-term therapeutic efficacy of phacoemulsification with intraocular 
lens implantation in patients with phacomorphic glaucoma. J Cataract Refract Surg 2010; 36: 783-9.

2- Mansouri $M$ et al. Anterior segment optical coherence tomography parameters in phacomorphic angle closure and mature cataracts. Invest Ophthalmol Vis Sci 2014; 55 (11): 7403-9.

3- Muzyka-Wozniak $M$ and Ogar A. Anterior chamber depth and iris and lens position before and after phacoemulsification in eyes with a short or long axial length. J Cataract Refract Surg. 2016; 42: 563-568.

4- Zhang Q, Jin W and Wang Q. Repeatability, reproducibility, and agreement of central anterior chamber depth measurements in pseudophakic and phakic eyes: optical coherence tomography versus ultrasound biomicroscopy. J Cataract Refract Surg. 2010; 36: 941-946.

5- Anayol, $\mathbf{M}$ et al. Comparison of central corneal thickness, thinnest corneal thickness, anterior chamber depth, and simulated keratometry using Galilei, Pentacam, and Sirius devices. Cornea. 2014; 33 (6): 582-586.

6- Rekas M, Barchan-Kucia K, Konopinska J, Mariak $\mathbf{Z}$ and Zarnowski T. Analysis and modling of anatomical changes of the anterior segment of the eye after cataract surgery with consideration of different phenotypes of eye structure. Current Eye Research. 2015; 40 (10): 1018-1027.

7- Nasser C, Singer R, Barkana Y, Zadok D, Avni I and Goldich Y. Repeatability of the Sirius imaging system and agreement with the pentacam HR. J Refract Surg. 2012; 28 (7): 493-497.
8- De La Parra-Colin P, Garza-Leon $M$ and Barrientos-Gutierrez T. Repeatability and comparability of anterior segment biometry obtained by the Sirius and the pentacam analysers. Int Ophthalmol. 2014; 34 (1): 27-33.

9- Lin YW, Wang TH, Hung PT. Biometric study of acute primary angle-closure glaucoma. J Formos Med Assoc. 1997; 96: 908-912.

10-Qi Y. Ultrasonic evaluation of the lens thickness to axial length factor in primary closure angle glaucoma. Yan Ke Xue Bao. 1993; 9: 12-14.

11-Altan C, Bayraktar S, Altan C, Eren H and Yilmaz OF. Anterior chamber depth, iridocorneal angle width, and intraocular pressure changes after uneventful phacoemulsification in eyes without glaucoma and with open iridocorneal angles. J Cataract Refact Surg. 2004; 30: 832-838.

12-Huang G, Gonzalez E, Lee R, Chen YC, He M and Lin SC. Association of biometric factors with anterior chamber angle widening and intraocular pressure reduction after uneventful phacoemulsification for cataract. J Cataract Refract Surg. 2012; 38: 108-116.

13-Pereira FA and Cronemberger S. Ultrasound biomicroscopic study of anterior segment changes after phacoemulsification and foldable intraocular lens implantation. Ophthalmology. 2003; 110: 1799.

14-Wang B, Sakata LM, Friedman DS, Chan YH, He M, Lavanya $R$, et al. Quantitative iris parameters and association with narrow angles. Ophthalmology. 2010; 117: 11-17.

awnallah, M., Salamah, M., Shawki, M., Solaiman, K. Changes in Anterior Chamber Depth and Angle Before and After Phacoemulsification of Intumescent Cataract. Zagazig University Medical Journal, 2019; July. 2020 Volume 26 Issue 4 (663-668): -. doi: 10.21608/zumj.2019.15051.1360 